\title{
Effect of short-term formaldehyde fixation on Raman spectral parameters of bone quality
}

Imke A.K. Fiedler

Michele Casanova

Tobias Keplinger

Björn Busse

Ralph Müller 


\title{
Effect of short-term formaldehyde fixation on Raman spectral parameters of bone quality
}

\author{
Imke A.K. Fiedler, ${ }^{a, b, \dagger}$ Michele Casanova, ${ }^{a, \dagger}$ Tobias Keplinger, ${ }^{c}$ Björn Busse, ${ }^{b}$ and Ralph Müller ${ }^{a, \star}$ \\ ${ }^{a}$ ETH Zurich, Institute for Biomechanics, Zurich, Switzerland \\ bUniversity Medical Center Hamburg-Eppendorf, Department of Osteology and Biomechanics, Hamburg, Germany \\ ${ }^{\mathrm{C} E T H}$ Zurich, Institute for Building Materials, Zurich, Switzerland
}

\begin{abstract}
Medical knowledge of the skeleton including its structures has improved constantly over the past decades. Advanced imaging methods, mechanical testing and optical techniques have revealed insights into bone architecture and composition. Most of these advancements were possible due to the ex vivo investigation of biological tissues. Investigations of fresh tissue are generally preferred over preserved or fixed samples. However, chemical fixation is sometimes inevitable due to histological procedures or logistical reasons. The aim of this study was to investigate whether short-term chemical fixation with formaldehyde affects bone quality parameters obtained from Raman spectroscopy and if these effects last for intermediate sample storage of several hours. As formaldehyde induces cross-links to the organic components in bone tissue, we hypothesized that collagen-related parameters are particularly affected. Femurs of eight 17 -week-old C57BL/6 mice were extracted and divided into two groups ( $N=8 /$ group). Samples of the first group were fixed by immersion in $4 \%$ formaldehyde (PFA-solution) for $12 \mathrm{~h}$ at $4^{\circ} \mathrm{C}$ (fixed group) while samples of the second group were left untreated (unfixed group). Raman spectroscopy was performed, and repeated after $4 \mathrm{~h}$, to assess whether intermediate storage time influenced the obtained results. Based on resultant spectra, mineral-to-matrix ratio, carbonate-to-phosphate ratio, carbonate-to-amide I ratio, mineral crystallinity and collagen maturity were determined. Carbonate-to-phosphate ratio was the only parameter showing a significant difference between the first and the subsequent measurements. For both groups, ratios showed a decrease in carbonate substitution compared to the first measurement (percentage decrease: $3.1 \%$ in fixed, $4.7 \%$ in unfixed). Collagen maturity of samples, which were short-term fixed with formaldehyde, was significantly lower than of fresh, unfixed samples (percentage difference: 3.8\%). Our study shows that Raman spectroscopy is able to detect changes in collagen structure initiated by formaldehyde and that changes in short-term fixed samples are minimally influencing bone material properties measured with Raman spectroscopy. @ The Authors. Published by SPIE under a Creative Commons Attribution 3.0 Unported License. Distribution or reproduction of this work in whole or in part requires full attribution of the original publication, including its DOI. [DOI: 10.1117/1.JBO.23.11.116504]
\end{abstract}

Keywords: Raman spectroscopy; bone quality; bone; fixation; PFA; paraformaldehyde; formaldehyde; collagen cross-linking.

Paper 180365R received Jun. 18, 2018; accepted for publication Nov. 5, 2018; published online Nov. 29, 2018.

\section{Introduction}

Raman spectroscopy has been established as a powerful tool for bone characterization by means of identifying molecule-specific vibrational modes of the sample's chemical components. ${ }^{1,2}$ In contrast to biomechanical testing, Raman spectroscopy requires only little sample preparation and allows nondestructive examination of bone material properties. Therefore, it is of great interest to find correlations between bone strength and bone quality parameters derived from Raman spectroscopy. ${ }^{3-6}$

Current research on the material properties of bone is therefore progressively pointing toward a multimodal approach, i.e., performing several subsequent analyses on the same samples. ${ }^{7-9}$ Such comprehensive studies usually encounter the problem of natural degeneration during the time samples are exposed to room temperature. Chemical fixation is a commonly performed procedure to hinder degenerative processes and hence to preserve the ultrastructure of biological tissue. The choice of the fixation protocol is not a trivial one and is essential for the outcome of an experiment, as the fixation process might alter certain tissue properties. ${ }^{10}$ The most commonly used fixative

*Address all correspondence to Ralph Müller, Email: ram@ethz.ch

tThese authors equally contributed to the work. is formaldehyde. This agent preserves biological tissue by cross-linking proteins present in the sample. In the literature, a great number of different fixation protocols applying formaldehyde-solutions are found. Formaldehyde is derived from paraformaldehyde (PFA) and is therefore also referred to as PFAsolution. Saturated formaldehyde solution is termed formalin and often stabilized with methanol. Variations in $\mathrm{pH}$, temperature, and fixation time of a protocol will result in fixed samples with different degrees of cross-linking. ${ }^{11}$ Depending on the laboratory and the purpose of investigation, typical fixation times can greatly vary. A few hours of fixation are commonly applied in immunohistochemistry and histology to preserve protein and cell structures. ${ }^{12}$ Weeks of fixation are common for intermediate storage between measurements, and months and years of fixation are performed for embalmment and long-term preservation. ${ }^{13}$

In the context of vibrational spectroscopy, bone tissue provides the signals of a composite material consisting of inorganic mineral crystals (mainly carbonated hydroxyapatite ${ }^{14}$ ) and an organic matrix (mainly type I collagen ${ }^{15}$ ). Employing Raman spectroscopy, it has been shown that chemical fixation of ex vivo bone samples with glycerol and ethanol significantly influences mineral-to-matrix ratio and crystallinity. ${ }^{16}$ Recently, it was reported that bone biopsies from human cadavers, which have 
been long-term embalmed in a formalin-ethanol solution also express an altered carbonate-to-phosphate ratio and collagen maturity assessed with Raman spectroscopy. ${ }^{17}$ Furthermore, it was found that fixation for $24 \mathrm{~h}$ with ethanol and formaldehyde irreversibly affects bone anisotropy by altering the organic matrix. ${ }^{18}$ Yet, whether bone tissue fixation of several hours with formaldehyde also has an effect on bone quality parameters determined by Raman spectroscopy is unreported.

This study intends to provide insights on sample preparation techniques for future experiments with bone specimens. Our aim is to investigate whether short-term chemical fixation with formaldehyde has an influence on bone quality parameters obtained from Raman spectroscopy and thus to show if Raman spectroscopy is sufficiently sensitive to detect these alterations. Due to the cross-linking effect of formaldehyde, we hypothesize that collagen-related bone quality parameters obtained from Raman spectroscopy are specifically susceptible to fixationinduced changes. Moreover, we want to verify whether Raman assessment of bone parameters is reproducible in fresh bones after several hours of storage at room temperature (i.e., simulating the exposure time of the samples when multiple experiments are performed). Similarly, to assess if fixed samples could deliver reproducible values after short-term storage, fixed samples were also remeasured after the same time elapsed for unfixed samples.

\section{Materials and Methods}

Eight 17-week-old C57BL/6 female mice were used in this study (Laboratoy Animal Facility at ETH, Zürich, Switzerland). All 16 femurs were extracted and eight independent femurs were randomly assigned to a group which underwent fixation (fixed group) while respective contralateral femurs were assigned to a group which remained unaltered (unfixed group). Both groups consisted of four right and four left femurs from the eight animals. All animal procedures were approved by the local authorities (Kantonales Veterinäramt Zürich, Zürich, Switzerland) and were performed in accordance with the approved regulations.

\subsection{Sample Preparation}

Samples of the fixed group were preserved by immersion in $3 \mathrm{ml}$ fixative immediately after dissection and stored for $12 \mathrm{~h}$ at $4^{\circ} \mathrm{C}$. Formaldehyde fixative was freshly prepared and was composed of a $4 \%$ phosphate-buffered paraformaldehyde-solution without the addition of methanol as stabilizer. Samples of the unfixed group did not undergo any treatment and were prepared for the measurements directly after dissection. Prior to the measurements, all samples underwent an identical sample preparation protocol to provide plane bone surfaces for standardized Raman spectroscopy measurements. Femoral heads were clipped off and the anterior distal parts of the femur were flattened. Subsequently, femurs were glued with the anterior side onto a microscope slide with a two-component epoxy resin adhesive (UHU Plus Schnellfest, UHU GmbH \& Co KG, Bühl, Germany). Grinding and polishing of the samples were then performed and standardized in all steps using increasing grades of carbide papers (P500, P1200, P2500, and P4000) and phosphate-buffered saline (PBS) as a coolant, until reaching the central longitudinal portion of the femur. A custom-built sample preparation stage was designed to ensure that the central portion was reached independently of the size of the sample.

\subsection{Raman Spectroscopy Settings}

All measurements were performed at room temperature with a confocal Raman spectroscope (Renishaw inVia, Renishaw plc, Wotton-under Edge, United Kingdom). The instrument was functioning with a red polarized-laser with a wavelength of $633 \mathrm{~nm}$. Dynamic line-scan imaging (Renishaw StreamLine mode) was used, where the laser is shaped into a line while automated, stepwise lateral movement of the sample stage along the $y$-axis (direction parallel to the streamline) enables equidistant pixel read-out along the line. Each line was set to $100 \mu \mathrm{m}$ and lateral step size was $1 \mu \mathrm{m}$, thus each line consisted of 101 readout points (see Fig. 1). An immersion objective (LUMPLFLN 60XW, Olympus Europa SE \& Co. KG, Hamburg, Germany) with $60 \times$ magnification and a numerical aperture (NA) of 1.0 resulted in a laser line width of $<1 \mu \mathrm{m}$, and allowed spectral acquisition while keeping samples hydrated in PBS. Exposure time was set to $120 \mathrm{~s}$ per line and a laser power measurement performed below the objective lens resulted in a power of $8.5 \mathrm{~mW}$.

\subsection{Spectral Acquisitions}

Seven lines for both lateral and medial cortical bone were acquired for each sample, whereby the first two line scans were performed in order to reduce photobleaching effects and therefore not included in the evaluation, leading to a total of 505 acquired spectra. Lines were located next to each other with an offset of $5 \mu \mathrm{m}$.

Measurements were performed directly after completion of sample preparation $\left(t_{0}\right)$ and spanned in total over a period of

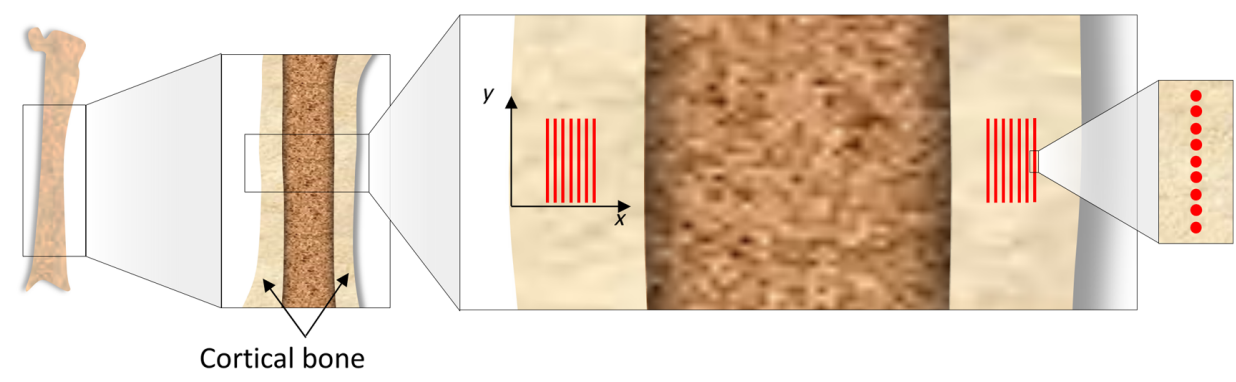

Fig. 1 Location and orientation of spectral acquisitions on cortical bone. Measurements were conducted in the mid-diaphysis ( $48 \%$ to $52 \%$ of the femur) on both medial and lateral sides of the samples. A total of seven lines with a length of $100 \mu \mathrm{m}$ and distance of $5 \mu \mathrm{m}$ were acquired, consisting of 101 single spectra per line. 


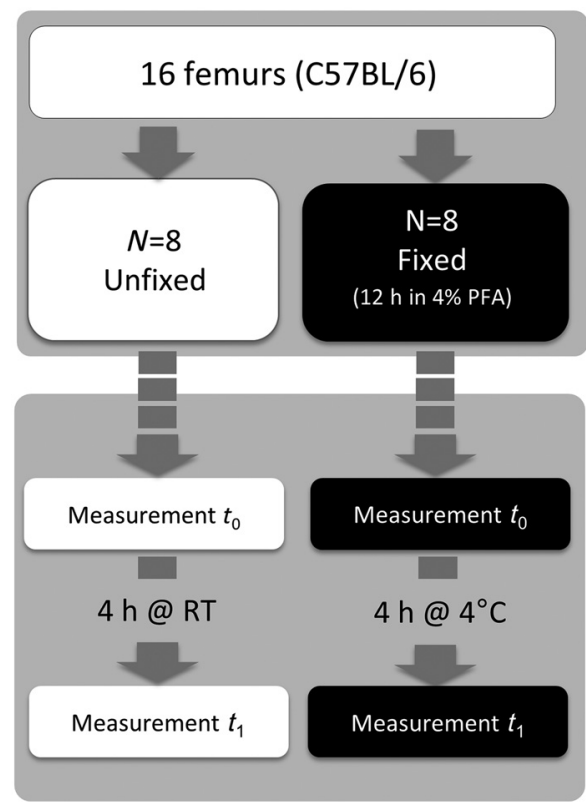

Fig. 2 A scheme representing the study. Sixteen femurs of eight B6 mice were divided in two groups (unfixed and fixed). Samples of the fixed group underwent 12 -h fixation in $4 \%$ PFA-derived formaldehyde. Raman spectroscopy was then performed on all samples directly after preparation at $t_{0}$. Four hours after completion of the experiments, measurements were repeated at $t_{1}$.

$2 \mathrm{~h}$ per sample. Samples were then stored for $4 \mathrm{~h}$ and measured a second time $6 \mathrm{~h}$ after the start of the first set of measurements $\left(t_{1}\right)$. Measurements at time $t_{1}$ were performed with an offset of $\sim 100 \mu \mathrm{m}$ in respect to measurements at time $t_{0}$ in order to avoid the excitation of the same area. Figure 1 shows the regions of the femur where Raman spectra were acquired.

During storage, samples of the unfixed group were kept hydrated in PBS-soaked gauze at room temperature to stimulate enhanced degradation, while samples of the fixed group were stored at air in a moist, dark chamber at $4^{\circ} \mathrm{C}$. A schematic representation of the measurements is shown in Fig. 2. Postprocessing of spectra was performed using Renishaw's Raman Environment software (WiRE, version 4.1, Renishaw plc, Gloucestershire, United Kingdom), Cytospec (v.2.00.01, Berlin, Germany), and a custom-written MATLAB ${ }^{\circledR}$ script (version R2014a, MathWorks, Inc., Natick, Massachusetts). Single spectra were averaged along one line and were then baseline corrected with an intelligent polynomial procedure implemented in WiRE 4.1. For determining subpeaks in the amide I envelope, an interactive peak fitting program was used (Peak Fitter, Version 3.6, Tom O'Haver, University of Maryland College Park, Maryland).

\subsection{Bone Quality Parameters}

For the analysis of the bone quality parameters, mineral-tomatrix ratio (area of phosphate/[area proline + area hydroxyproline]), crystallinity (inverse of the full-width-at-half-maximum, $\mathrm{FWHM}^{-1}$, of the phosphate peak), carbonate-to-amide I ratio (area of carbonate/area of amide I), carbonate-to-phosphate ratio (area of carbonate/area of phosphate), and collagen maturity (area of amide I subpeak around $1660 \mathrm{~cm}^{-1} /$ area of subpeak around $1690 \mathrm{~cm}^{-1}$ ) were derived as previously described. ${ }^{19}$ Peak intervals are shown in Fig. 3.

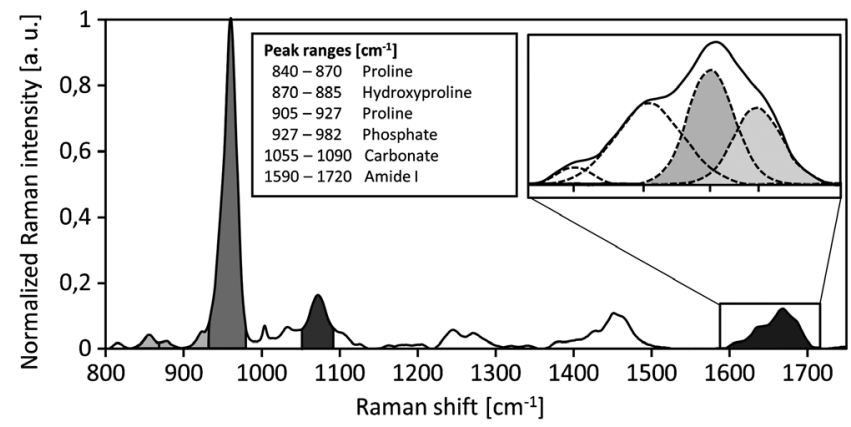

Fig. 3 Raman spectrum of mouse bone and chosen peak intervals. Bone quality parameters were determined using an in-house $M A T L A B^{\circledR}$ script. The different parameters were derived from analyses of the curves and areas under the curves.

\subsection{Statistical Analysis}

For all statistical analyses, IBM SPSS Statistics ${ }^{\circledR}$ (Version 22, IBM Corporation, New York) was used. Normal distribution and homogeneity of variances were tested with the KruskalWallis test and Levene's test, respectively. Since no significant differences were found between lateral and medial values of each sample ( $p>0.05$ for all parameters, paired $t$-test), data were averaged. ANOVA with repeated measures was then conducted between groups. Hereby, time was considered as a within-subjects factor consisting of two levels (i.e., $t_{0}$ and $t_{1}$ ), while group (i.e., unfixed and fixed) was considered as a between-subjects factor. Significance values and main effects were determined for time, group, and their interaction at a significance level of $\alpha=0.05$.

\section{Results}

For mineral-to-matrix ratio, crystallinity and carbonate-to-amide I ratio, no significant differences between groups (unfixed versus fixed) and within groups ( $t_{0}$ versus $\left.t_{1}\right)$ were found. Mineralto-matrix ratio in the unfixed group resulted in $14.00 \pm 0.83$ at $t_{0}$ and $14.02 \pm 0.63$ at $t_{1}$; in the fixed group in $14.32 \pm 0.96$ at $t_{0}$ and $14.34 \pm 0.76$ at $t_{1}$. Crystallinity yielded in the unfixed group $0.0568 \pm 0.0004$ at $t_{0}$ and $0.0567 \pm 0.0005$ at $t_{1}$; in the fixed group $0.0570 \pm 0.0006$ at $t_{0}$ and $0.0568 \pm 0.0006$ at $t_{1}$. Carbonate-to-amide $\mathrm{I}$ ratio resulted in the unfixed group in $0.817 \pm 0.068$ at $t_{0}$ and $0.789 \pm 0.058$ at $t_{1}$ and in the fixed group in $0.783 \pm 0.056$ at $t_{0}$ and $0.789 \pm 0.052$ at $t_{1}$.

Table 1 shows the percentage changes in Raman bone parameters between the time point $t_{0}$ and $t_{1}$ within the fixed and unfixed groups, and the percentage differences in Raman bone parameters between the fixed and unfixed groups at both time points $t_{0}$ and $t_{1}$.

Carbonate-to-phosphate ratio [Fig. 4(a)] showed a small but significant decrease within both groups between measurement at $t_{0}$ and $t_{1} \quad(p<0.001)$, whereby the fixed group registered a smaller percentage decrease from $0.221 \pm 0.005$ to $0.215 \pm 0.003(-3.1 \%)$ than the unfixed group from $0.222 \pm$ 0.008 to $0.212 \pm 0.007(-4.7 \%)$. An influence of short-term fixation on the carbonate-to-phosphate ratio could not be detected between the two groups $(p=0.659)$ and the interaction of both factors was insignificant $(p=0.171)$.

The value of the collagen maturity parameter [Fig. 4(b)] was significantly lower in the fixed group than in the unfixed group for both time points, with $1.328 \pm 0.059$ versus $1.405 \pm 0.051$ at $t_{0}$ and $1.346 \pm 0.052$ versus $1.405 \pm 0.039$ at $t_{1}(p=0.007)$. 
Table 1 Percentage changes of bone parameters over time within fixed and unfixed groups and percentage differences in bone parameters between fixed and unfixed groups per time point $(N=8 /$ group). Abbreviations: mineral-to-matrix ratio (M:M), carbonate-to-amide I ratio (C:Aml), and carbonate-to-phosphate ratio (C:P). Significant differences with $p<0.05$ are indicated $\left.{ }^{*}\right)$ for within-subjects factor (time) and for betweensubjects factor (group).

\begin{tabular}{|c|c|c|c|c|c|c|}
\hline \multirow[b]{2}{*}{ Raman bone parameter } & \multicolumn{3}{|c|}{ Percentage change between time point $t_{0}$ and $t_{1}$} & \multicolumn{3}{|c|}{$\begin{array}{l}\text { Percentage difference between unfixed and } \\
\text { fixed groups }\end{array}$} \\
\hline & $\begin{array}{l}\text { Unfixed } \\
\text { group }\end{array}$ & $\begin{array}{l}\text { Fixed } \\
\text { group }\end{array}$ & $\begin{array}{c}p \text {-Value } \\
\text { (within-subjects } \\
\text { factor: time) }\end{array}$ & $\begin{array}{l}\text { At time } \\
\text { point } t_{0}\end{array}$ & $\begin{array}{l}\text { At time } \\
\text { point } t_{1}\end{array}$ & $\begin{array}{c}p \text {-value } \\
\text { (between-subjects } \\
\text { factor: group) }\end{array}$ \\
\hline M:M (-) & $0.1 \%$ & $0.1 \%$ & 0.993 & $1.5 \%$ & $1.5 \%$ & 0.380 \\
\hline Crystallinity (-) & $-0.2 \%$ & $-0.4 \%$ & 0.374 & $0.2 \%$ & $0.1 \%$ & 0.632 \\
\hline C:Aml (-) & $-3.4 \%$ & $0.8 \%$ & 0.502 & $-2.9 \%$ & $0.0 \%$ & 0.517 \\
\hline$C: P(-)$ & $-4.7 \%$ & $-3.1 \%$ & $<0.001^{*}$ & $-0.3 \%$ & $0.8 \%$ & 0.659 \\
\hline Collagen maturity $(-)$ & $0.0 \%$ & $1.3 \%$ & 0.517 & $-3.8 \%$ & $-2.9 \%$ & $0.007^{*}$ \\
\hline
\end{tabular}
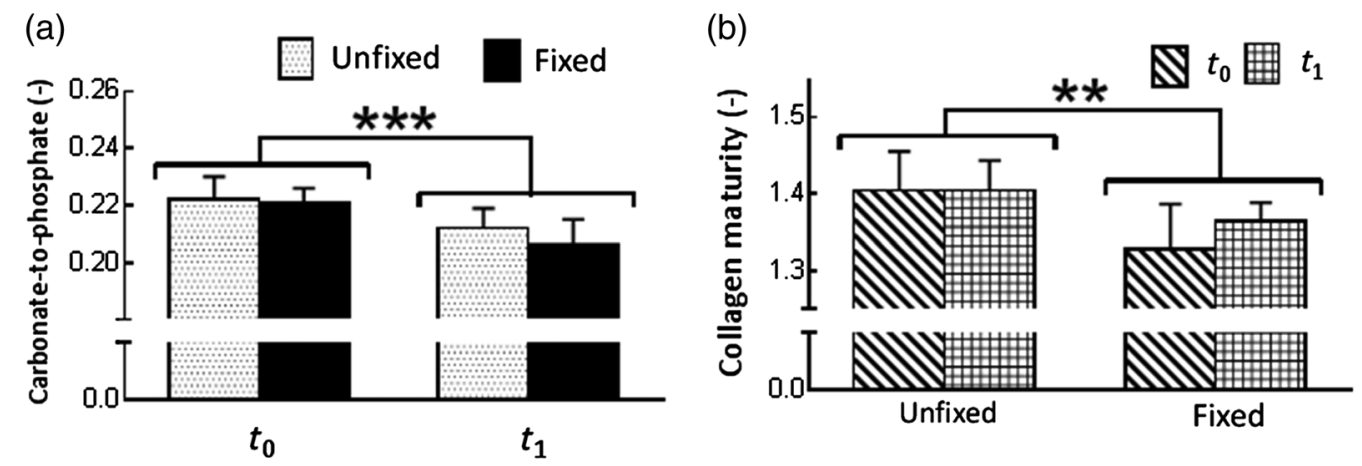

Fig. 4 (a) Carbonate-to-phosphate ratio and (b) collagen maturity of fixed and unfixed groups at the two different time points. A repeated measures ANOVA showed a significant difference over time in carbonate-to-phosphate ratio within both groups $(p<0.001)$, and a difference in collagen maturity parameter between the groups $(p<0.01)$.

The percentage difference between both groups was $3.8 \%$ at time $t_{0}$ and $2.9 \%$ at time $t_{1}$. An influence of time was not found within both groups $(p=0.517)$, although the fixed group registered a slight increase by $1.3 \%$ over time. An interaction between both factors was not detected $(p=0.518)$.

\section{Discussion}

In this study, we investigated whether short-term chemical fixation with formaldehyde has an influence on bone quality parameters obtained from Raman spectroscopy. Moreover, we aimed to verify if any changes are observable after a period of several hours at room temperature compared to an unfixed group. The evaluation of bone quality parameters supported the hypothesis that fixation with formaldehyde predominantly affects parameters which are associated with the composition of collagen. Although previous studies investigating the effects of long-term fixation have shown no influence on collagen maturity, in our study, this parameter was indeed the only parameter significantly affected by the short-term fixation over $12 \mathrm{~h}$ with $4 \%$ formaldehyde.

The collagen maturity parameter expressed as ratio between the amide I subpeaks at 1660 and $1690 \mathrm{~cm}^{-1}$ was first derived with Fourier transform infrared spectroscopy (FTIR) by
Paschalis et al. ${ }^{20}$ It has been assumed to represent the ratio of mature pyridinolines (PYD) to immature dihydroxylysinorleucine (DHLNL) and has therefore been correlated to the quantity of cross-links present in collagen proteins of bone. ${ }^{20}$ This has been adopted by Raman spectroscopy as a gold standard for determining collagen cross-links. For the past years, the interpretation has been under debate due to different conclusions also attained with FTIR. ${ }^{21,22}$ A recent publication by Paschalis et al. then confirmed that the peak at $\sim 1660 \mathrm{~cm}^{-1}$ is well correlated with PYD. ${ }^{23}$ But no confirmation about the validity of the detection of the DHLNL peak at $\sim 1690 \mathrm{~cm}^{-1}$ was expressed. In the future, we expect further examinations on the number and position of subpeaks in the amide I envelope in order to fully understand to which molecules they are associated. However, it seems to be unquestionable that mutations in this envelope correlate with changes to the collagen secondary structure. ${ }^{4}$

In our study, the chemical reactions of formaldehyde with proteins of the samples seemed to change their structure and thereby to decrease the collagen maturity parameter. It should be noted that a fixation protocol for immunohistochemical applications with a fixation time of $12 \mathrm{~h}$ was chosen. This rather short fixation time was proven to be sufficient to cause significant changes in the collagen secondary structure. Formaldehyde 
functions in two steps: fast diffusion and slow fixation. ${ }^{24}$ It seems commonly accepted that a firm bonding of formaldehyde occurring in prolonged fixation results in irreversible crosslinks. ${ }^{24}$ Hence, it is most likely that $12 \mathrm{~h}$ of fixation were sufficient for formaldehyde to penetrate into the tissue, thereby changing its molecular structure by means of initiating instable cross-links leading to a decrease in the ratio $\left(1660 / 1690 \mathrm{~cm}^{-1}\right)$. Subsequent unpublished investigations on the stability of these formaldehyde-induced cross-links showed that the collagen maturity parameter of fixed samples after $t_{1}$ could again be increased by $1 \%$ after $24 \mathrm{~h}$ of washing the samples in PBS (i.e., regained values similar to the initial measurement at $t_{0}$ ). Thus, the increase of this parameter in the fixed group between $t_{0}$ and $t_{1}$ could be explained by the fact that the samples were covered with PBS during the first acquisition. Finding a physicochemical and biological description of processes leading to the induced alterations goes beyond the scope of this study, as actual events during diffusion and cross-linking are poorly understood.

A decrease in carbonate-to-phosphate ratio was observed at time $t_{1}$ in both fixed and unfixed groups, whereby the effect was observed to a lesser extent in the fixed group. It is not clear whether the decrease is a consequence of natural degeneration over time, or of storage and measuring in PBS, or of chemical effects due to repeated laser excitation of the sample. To reduce occurrence of tissue alterations caused by repeated exposure to the laser on the regions of interest, Raman line laser measurements were performed with an offset of $100 \mu \mathrm{m}$. Yet, this small change in laser position may have influenced the measurement outcomes between time point $t_{0}$ and $t_{1}$. Further, the specimens of the unfixed group underwent a different storage setting than the fixed group between the measurement time points in order to stimulate enhanced progression of natural degeneration. As degeneration occurs faster at higher temperatures (RT versus $4^{\circ} \mathrm{C}$ ), this may have caused more pronounced differences in the carbonate-to-phosphate ratio in the unfixed group. As unbuffered formaldehyde is known to initiate mineral dissolution, ${ }^{25}$ $\mathrm{pH}$ level was thoroughly controlled during this study. Both unfixed and fixed samples were covered with PBS for $\sim 2 \mathrm{~h}$ per time step during spectra acquisitions. It is possible that excessive amounts of phosphate in the samples' environment led to a higher presence of this compound in the bone matrix and thus to a decrease in the carbonate-to-phosphate ratio. A higher decrease in unfixed samples could then be explained with their permanent hydration with PBS-soaked gauze, whereas fixed samples were kept in a moist chamber for avoiding defixation due to the contact with PBS. However, keeping bone tissue in PBS is a standard method to prevent bone degeneration during sample handling. ${ }^{26}$ Since the determined phosphate-related bone quality parameters crystallinity and mineral-to-matrix ratio did not show clear alterations after short-term storage, the interpretation of detected differences in the carbonate-to-phosphate parameter is rendered rather complex.

Concerning the laser exposure of the specimens, high intensities may lead to burning of irradiated regions. To avoid such effects, line-shaped laser illumination was used in our setup. Hereby, the laser intensity of a spot is distributed onto a line, leading to a reduction of potential laser-induced damage. Indeed, to avoid the occurrence of burning, the measured laser intensity in this study was kept at a minimum compared to previous reports on bone. ${ }^{27,28}$ In contrast to point-by-point imaging using spot illumination [i.e., two-dimensional (2-D) mapping], line imaging may require higher exposure times in order to gain similar signal-to-noise ratios, generally leading to longer acquisition times. One major advantage of 2-D mapping is that it can be employed to provide spatial information at high resolution. This has been demonstrated with regard to intermatrix-alterations in bone at the tissue level ${ }^{29}$ as well as intercellular heterogeneity, e.g., in fibroblasts at the cellular level. ${ }^{30}$ In this study, all spectra acquired along one line were averaged to one spectrum, leading to a loss of such spatial information of the bone quality parameters, but to an increase in signal-to-noise ratio without increasing the risk of burning the fresh and hydrated samples.

In contrast to previously reported Raman measurements gained from cadaveric bone biopsies, which were embalmed long-term in an ethanol-formalin solution, ${ }^{17}$ the mineral-related parameters determined in our study did not appear to be affected by the $12 \mathrm{~h}$-fixation in formaldehyde. In addition to the difference in fixation time, this could be linked to the different composition of the fixation media applied, which included ethanol and possibly methanol as stabilizer in contrast to the PFAsolution applied in this study.

With regard to the mineral-to-matrix ratio, it should be noted that several different peak ratios have been reported in the literature, including phosphate-to-amide III ratio and the most traditional peak phosphate-to-amide I ratio. ${ }^{4,31}$ It has been suggested that the intensity of certain collagen-related peaks including amide $\mathrm{I}$ is dependent on the orientation of collagen fibers with respect to the incident beam. In this study, we used hydroxyproline and proline to determine the mineral-to-matrix ratio, which appears to be less prone to this effect. ${ }^{4,32}$ Moreover, Taylor et al. have recently validated a number of Raman-derived mineral-tomatrix ratios against thermal gravimetric analysis, supporting the use of the phosphate and hydroxyproline + proline peaks to assess mineral content. ${ }^{31}$ Considering these aspects, we suspect that the similar results of the mineral-to-matrix ratio between the unfixed and fixed groups are indeed due to negligible influence of short-term formaldehyde fixation on the intensity of these peaks.

\section{Conclusions}

This study investigated the influence of short-term fixation with formaldehyde on bone quality parameters frequently obtained from Raman spectroscopy and their reproducibility after a period of several hours of exposure to room temperature (unfixed group) and preservation at $4^{\circ} \mathrm{C}$ (fixed group).

Results showed a significant decrease of the carbonate-tophosphate ratio after intermediate storage for both fixed and unfixed bones. When aiming at the evaluation of carbonate substitution, the comparison of bone samples that were exposed to buffer for different amounts of time or had undergone multiple measurements might lead to spurious results.

The collagen-related ratio around $1660 \mathrm{~cm}^{-1} / 1690 \mathrm{~cm}^{-1}$ was the only parameter significantly affected by the 12-h fixation protocol used within this study. We demonstrated that Raman spectroscopy is able to detect even small changes within the collagen structure caused by fixation with formaldehyde. It is important to note that these conclusions only apply to short-term fixation, e.g., protocols ideal for immunohistochemistry. Whether longer formaldehyde fixation of bone samples for days or weeks (often performed in laboratory practice) would lead to comparable results cannot be answered with the results 
of this study and requires further investigation with regard to the time-dependency of bone sample fixation with fresh and nonstabilized formaldehyde. Yet, some suggestions for future bone analysis using Raman spectroscopy can be derived: (i) bone quality parameters gained from mouse femurs can be pooled regardless the anatomical origin (i.e., lateral or medial site), (ii) variations in storage times $>2 \mathrm{~h}$ between measurements may be critical when the carbonate-to-phosphate parameter is of particular interest, and (iii) bone specimens that have undergone identical short-term formaldehyde fixation can be compared and/or pooled in Raman analyses.

\section{Disclosures}

The authors declare no conflict of interest.

\section{Acknowledgments}

The authors would like to thank Bastien Chatton for technical assistance.

\section{References}

1. H. B. Hunt and E. Donnelly, "Bone quality assessment techniques: geometric, compositional, and mechanical characterization from macroscale to nanoscale," Clin. Rev. Bone. Miner. Metab. 14(3), 133-149 (2016).

2. H. Kimura-Suda and T. Ito, "Bone quality characteristics obtained by Fourier transform infrared and Raman spectroscopic imaging," J. Oral Biosci. 59(3), 142-145 (2017).

3. E. P. Paschalis, S. Gamsjaeger, and K. Klaushofer, "Vibrational spectroscopic techniques to assess bone quality," Osteoporosis Int. 28(8), 2275-2291 (2017).

4. G. S. Mandair and M. D. Morris, "Contributions of Raman spectroscopy to the understanding of bone strength," Bonekey Rep. 4, 620 (2015).

5. K. Buckley et al., "Towards the in vivo prediction of fragility fractures with Raman spectroscopy," J. Raman Spectrosc. 46(7), 610-618 (2015).

6. X. Bi et al., "Raman and mechanical properties correlate at whole bone- and tissue-levels in a genetic mouse model," J. Biomech. 44(2), 297-303 (2011)

7. N. Rodriguez-Florez et al., "An investigation of the mineral in ductile and brittle cortical mouse bone," J. Bone Miner. Res. 30(5), 786-795 (2015).

8. M. Casanova et al., "Quantitative phenotyping of bone fracture repair: a review," Bonekey Rep. 3, 550 (2014).

9. E. Donnelly, "Methods for assessing bone quality: a review," Clin. Orthop. Relat. Res. 469(8), 2128-2138 (2011).

10. G. Penel et al., "Composition of bone and apatitic biomaterials as revealed by intravital Raman microspectroscopy," Bone 36(5), 893-901 (2005).

11. S.-R. Shi, Y. Shi, and C. R. Taylor, "Antigen retrieval immunohistochemistry: review and future prospects in research and diagnosis over two decades," J. Histochem. Cytochem. 59(1), 13-32 (2011).

12. A. P. Kusumbe et al., "Sample preparation for high-resolution 3D confocal imaging of mouse skeletal tissue," Nat. Protoc. 10(12), 1904-1914 (2015).
13. E. Brenner, "Human body preservation: old and new techniques," J. Anat. 224(3), 316-344 (2014).

14. J.-D. P. McElderry et al., "Crystallinity and compositional changes in carbonated apatites: evidence from 31P solid-state NMR, Raman, and AFM analysis," J. Solid State Chem. 206 (2013).

15. S. Ranjit et al., "Imaging fibrosis and separating collagens using second harmonic generation and phasor approach to fluorescence lifetime imaging," Sci. Rep. 5, 13378 (2015).

16. Y. N. Yeni et al., "Effect of fixation and embedding on Raman spectroscopic analysis of bone tissue," Calcif. Tissue Int. 78(6), 363-371 (2006).

17. T. Pascart et al., "Bone samples extracted from embalmed subjects are not appropriate for the assessment of bone quality at the molecular level using Raman spectroscopy," Anal. Chem. 88(5), 2777-2783 (2016).

18. N. Hammer et al., "Ethanol and formaldehyde fixation irreversibly alter bones' organic matrix," J. Mech. Behav. Biomed. Mater. 29, 252-258 (2014).

19. M. D. Morris and G. S. Mandair, "Raman assessment of bone quality," Clin. Orthop. Relat. Res. 469(8), 2160-2169 (2011).

20. E. P. Paschalis et al., "Spectroscopic characterization of collagen crosslinks in bone," J. Bone Miner. Res. 16(10), 1821-1828 (2001).

21. E. M. B. McNerny et al., "Bone fracture toughness and strength correlate with collagen cross-link maturity in a dose-controlled lathyrism mouse model," J. Bone Miner. Res. 30(3), 455-464 (2015).

22. D. Farlay et al., "The ratio $1660 / 1690 \mathrm{~cm}(-1)$ measured by infrared microspectroscopy is not specific of enzymatic collagen cross-links in bone tissue," PLoS One 6(12), e28736 (2011).

23. E. P. Paschalis et al., "Fourier transform infrared spectroscopic characterization of mineralizing type I collagen enzymatic trivalent crosslinks," Calcif. Tissue Int. 96(1), 18-29 (2015).

24. H. Puchtler and S. N. Meloan, "On the chemistry of formaldehyde fixation and its effects on immunohistochemical reactions," Histochemistry 82(3), 201-204 (1985).

25. T. Asaka and H. Kikugawa, "Influence of preservation in two kinds of formaldehyde solutions on the fracture characteristics of bovine femoral compact bones," Mater. Trans. 48(1), 16-20 (2007).

26. Y. H. An and K. L. Martin, Handbook of Histology Methods for Bone and Cartilage, Humana Press, Totowa, New Jersey (2003).

27. S. Gamsjaeger, K. Klaushofer, and E. P. Paschalis, "Raman analysis of proteoglycans simultaneously in bone and cartilage," J. Raman Spectrosc. 45(9), 794-800 (2014).

28. J. A. Timlin et al., "Raman spectroscopic imaging markers for fatiguerelated microdamage in bovine bone," Anal. Chem. 72(10), 2229-2236 (2000).

29. S. Gamsjaeger et al., "Vibrational spectroscopic imaging for the evaluation of matrix and mineral chemistry," Curr. Osteoporosis Rep. 12(4), 454-464 (2014).

30. A. J. Hobro and N. I. Smith, "An evaluation of fixation methods: spatial and compositional cellular changes observed by Raman imaging," Vib. Spectrosc. 91, 31-45 (2017).

31. E. A. Taylor et al., "Raman and Fourier transform infrared (FT-IR) mineral to matrix ratios correlate with physical chemical properties of model compounds and native bone tissue," Appl. Spectrosc. 71(10), 2404-2410 (2017).

32. J. S. Nyman et al., "Measuring differences in compositional properties of bone tissue by confocal Raman spectroscopy," Calcif. Tissue Int. 89(2), 111-122 (2011).

Biographies of the authors are not available. 